\title{
Foam in Iron and Steelmaking
}

\author{
A. K. Lahiri, R. Yogambha ${ }^{*}$, Pranesh Dayal and S. Seetharaman' \\ Department of Metallurgy, Indian Institute of Science, Bangalore 560012. India \\ *Presently Center for Nano Technology and Bio-Materials, University of Queensland, Australia \\ Division of Metallurgy, Royal Institute of Technology, 100 44 Stockholm, Sweden
}

\begin{abstract}
Foam is common in iron and steelmaking processes. Iron and steelmaking slags have inherent foaminess but the presence of strong surface active compounds like $\mathrm{P}_{2} \mathrm{O}_{5}, \mathrm{Cr}_{2} \mathrm{O}_{3}, \mathrm{CaS}, \mathrm{V}_{2} \mathrm{O}_{5}$ and $\mathrm{CaF}_{2}$ make the foam more stable.

Stable foam can form only in the presence of surface active agents. When a bubble rises on the surface of a liquid, the film around it develops a concentration gradient of surface active agents such that Marangoni force acts in upward direction. This prevents liquid drainage from the film. The presence of surface active agents favours formation of smaller bubbles and prevents bubble rupture as well. So a strong surface active agent where $d \sigma / d c$ is high makes the foam very stable.

When a bubble comes into contact with a solid surface, the dynamic contact angle is different from the equilibrium value. If the former is less than the latter, there is a resultant outward force which tries to make the bubble flat. This force is responsible for the collapse of foamy slags in the presence of large carbonaceous particles. This force is not significant for very small bubbles and is not effective when carbon particles are much smaller than the bubble.
\end{abstract}

\section{INTRODUCTION}

The slag foaming phenomenon was well known to steel makers of Open Hearth furnaces. It prevented effective heat transfer from flame to bath and reduced the roof life. The techniques to control foam by adjusting slag chemistry, flame characteristics and throwing of coke were known to the Open Hearth operators. Since foaming phenomena are associated with the characteristics of steelmaking slag, it occurs in the BOF process as well. A foamy slag with a large number of fine metal droplets dispersed in it increases the reaction rates and thereby is helpful for the BOF process. But sometimes it leads to slopping, causing metal loss and interruption in converter operation. So control of foaming to prevent slopping is an important aspect in BOF operation. On the other hand, in electric arc furnaces, foamy slag practice is followed to improve the efficiency of the process. It shields the refractory from the arc, metal from the furnace atmosphere and helps to stabilise the arc. Foaming is found in hot metal pretreatment such as desiliconization, dephosphorization and desulphurisation of pig iron. In bath smelting processes, a foamy slag is essential. It permits rapid heat transfer to the metal from the post combustion and at the same time maintains reducing condition in the melt /13\%. In the blast furnace foamy slag can form in the dripping zone where iron oxide rich slag drips over the coke bed. Diao /4/ points out that foamy slag creates problems in smelting of vanadium bearing titanomagnetite in blast furnaces. So foaming is a part of iron and steel making processes and its control is essential for process optimisation.

The importance of understanding the foaming behaviour of slag was realized by metallurgists quite early. As early as 1959 Cooper and Kitcher /5/ measured the time required to decay a given volume of foam or foam life for $\mathrm{CaO}-\mathrm{SiO}_{2}$ slag. They found that decrease of temperature and basicity increased the foam life but a small addition of $\mathrm{P}_{2} \mathrm{O}_{5}$ significantly increased it. Swisher and McCabe /6/ reported that the addition of $\mathrm{Cr}_{2} \mathrm{O}_{3}$ in $\mathrm{CaO}-\mathrm{SiO}_{2}$ slag increased foam life. Hara et al. 
17/ found that the foam life, foam height and surface tension of slags are related for $\mathrm{CaO}-\mathrm{FeO}, \mathrm{SiO}_{2}-\mathrm{FeO}$ and $\mathrm{CaO}-\mathrm{SiO}_{2}-\mathrm{FeO}$ systems. In the last decade or so Fruehan and his associates /8-12/ systematically measured and correlated the foaming characteristics for different slags of importance to iron and steelmaking. To quantify the foaming characteristics, they measured foaming index defined as

$$
\Sigma=h / u_{5}
$$

where $\mathrm{h}$ is the height of the foam at steady state when gas with superficial velocity $u_{s}$ is passed through it. They correlated foaming index with slag characteristics and bubble diameter. Recently Lahiri and Seetharaman 113 showed that foaming index is given by

$$
\Sigma=C \mu_{s} /\left(\rho_{s l} d_{b}\right)
$$

where $\mu_{\mathrm{sl}}$ and $\rho_{\mathrm{sl}}$ are respectively the viscosity and density of slag and $d_{b}$ is the bubble diameter. $C$ is determined by gas fraction in the foamy slag, bubble shape and ratio of bulk to surface viscosity of slag. They further pointed out that foaming index is independent of gas flow rate only for two phase foam which has upper foamy layer and lower liquid layer with bubbles dispersed in it.

Ogawa et al. $/ 14,15 /$ studied the behaviour of foam in the presence of carbonaceous material, iron oxide pellets and alumina block by $\mathrm{X}$-ray fluoroscopy in slags containing $\mathrm{CaO}-\mathrm{SiO}_{2}-\mathrm{Al}_{2} \mathrm{O}_{3}$ and $\mathrm{FeO}$. They found that when large bubbles came in contact with a block of graphite, coke or glassy carbon, bubbles ruptured over its surface. The gas gathered on the block and finally a large bubble evolved from its top surface. But the bubbles in contact with iron ore pellets or alumina did not rupture. Zhang and Fruehen /16/ studied the effect of the addition of spherical carbonaceous particles, alumina and iron ore pellets in $\mathrm{CaO}-\mathrm{SiO}_{2}-\mathrm{CaF}_{2}$ and $\mathrm{CaO}-\mathrm{SiO}_{2}-\mathrm{Al}_{2} \mathrm{O}_{3}-\mathrm{CaF}_{2}$ slags. They found that when a bubble came in contact with a carbon particle, it tried to engulf it and in the process rose up along the particle and finally escaped. This led to foam collapse but alumina or iron pellets did not affect the foam. They explained the observation based on the mechanism proposed by Dipppenaar $/ 17 /$ who suggested that the difference between the instantaneous and equilibrium contact angle for non-wetting particles forces the bubble film to move rapidly toward the particle and stretch the film thin enough to rupture. Recently Wu et al. /18/ found that the addition of coke or coal fines in slag increased the foaminess. So there is a need to rationalize the above observations based on the mechanism of foam formation and rupture.

The present article aims at evaluating factors that determine the foaminess of slag and its collapse.

\section{FOAMINESS}

According to Gibbs adsorption isotherm, excess surface concentration of solute, $\Gamma_{s}$, is given by

$$
\Gamma_{\mathrm{s}}=-\mathrm{d \sigma} / \mathrm{RTd}[\ln (\mathrm{a})]
$$

where $\sigma$ is the surface tension of the solution and ' $a$ ' is the activity of solute. So the surface concentration of a surface active solute is higher and surface concentration of solutes, which increase the surface tension. is lower. In other words, the surface concentration of solutes is such that surface tension is lowest. When a bubble comes on the surface of a liquid. it carries a thin film of liquid along with it. The top surface of the bubble comes in contact with the liquid surface first. so the surface tension at the top of the film is lowest. In the downward direction along the height of the film. the solute concentration gradually moves towards the bulk concentration leading to an increase of surface tension. So a surface tension gradient is set up in the film around the bubble such that there is an upward force. This Marangoni force prevents fast draining of liquid from the film and thereby helps foam formation. Obviously, the draining rate decreases with increase of $\mathrm{d} \sigma / \mathrm{dc}$ where $c$ is the concentration of solute. Recently Nexhip $c t$ al. /19/ studied the effect of surface active agents on the film drainage rate and found that the film drainage rate is inversely related to $d \sigma / d c$. This observation supports the mechanism of the build up of concentration gradient of solute in the film. Since concentration of solute is zero in pure liquid, foam does not form in them. a fact 
first recognized by Plateau more than a hundred and thirty years ago.

Fig. 1 shows $/ 20 /$ the effect of different solutes on the surface tension of $\mathrm{CaO}-\mathrm{SiO}_{2}$ systems. All solutes shown in Fig. $\mathrm{I}$ are surface active except $\mathrm{Al}_{2} \mathrm{O}_{3}$. The data of $\mathrm{CaO}-\mathrm{Al}_{2} \mathrm{O}_{3}-\mathrm{SiO}_{2}$ system show that $\mathrm{SiO}_{2}$ is surface active for $\mathrm{CaO}-\mathrm{Al}_{2} \mathrm{O}_{3}$ system. However, in all cases surface tension is dependent on concentration and thereby it can be concluded that iron and steelmaking slags have inherent foaminess. Since $\mathrm{P}_{2} \mathrm{O}_{5}, \mathrm{Cr}_{2} \mathrm{O}_{3}, \mathrm{CaS}$, $\mathrm{V}_{2} \mathrm{O}_{5}$ and $\mathrm{CaF}_{2}$ are strongly surface active, the effect of these species is greater compared to $\mathrm{SiO}_{2}, \mathrm{Al}_{2} \mathrm{O}_{3}$ or $\mathrm{Fe}_{\mathrm{x}} \mathrm{O}$.

When a foam bubble tries to burst, a part of the film around the bubble must become thinner. The resistance for the thinning is surface viscosity of the film. So solutes, which increase the surface viscosity, increase the foaminess of slag. Due to some disturbances if a portion of bubble stretches, the concentration of solute becomes less in that portion. If the solute is surface active, the surface tension of the thinned portion becomes higher compared to surrounding surfaces. The higher surface tension tries to thicken the thinned portion or heal the injury. Thereby it can be inferred that the presence of surface active solutes increases the foaminess of slag or makes the foam stable. On the other hand, if $\mathrm{d} \sigma / \mathrm{dc}$ is positive, any disturbance will make the bubble burst so the stability of foam will be less. The observations of Hara et al. 121 , who found that stable foam forms when surface viscosity of melt is relatively high and melt contains surface active compounds, is in agreement with the above mechanism.

\section{FOAM STABILITY}

In general, the drainage of liquid through the Plateau border results in bubble rupture. Based on the above mechanism of foam rupture, Lahiri and Seetharaman $/ 13 /$ showed that foaming index is given by Eq. 2 . Since foaming index is inversely proportional to rate constant for foam decay $/ 13 /$, it is a measure of foam life or stability of foam. Eq. 2 shows that foaming index is inversely proportional to bubble size, thereby conditions that favour formation of smaller bubble result in higher foam height. Ogawa et al. 122/ calculated the bubble size formed at the slag metal interface by solving force balance equations. Their results show that bubble diameter increases linearly with the increase of surface tension of slag and contact angle. The surface active compounds $\mathrm{P}_{2} \mathrm{O}_{5}, \mathrm{Na}_{2} \mathrm{O}$ and $\mathrm{CaF}_{2}$ reduce both the

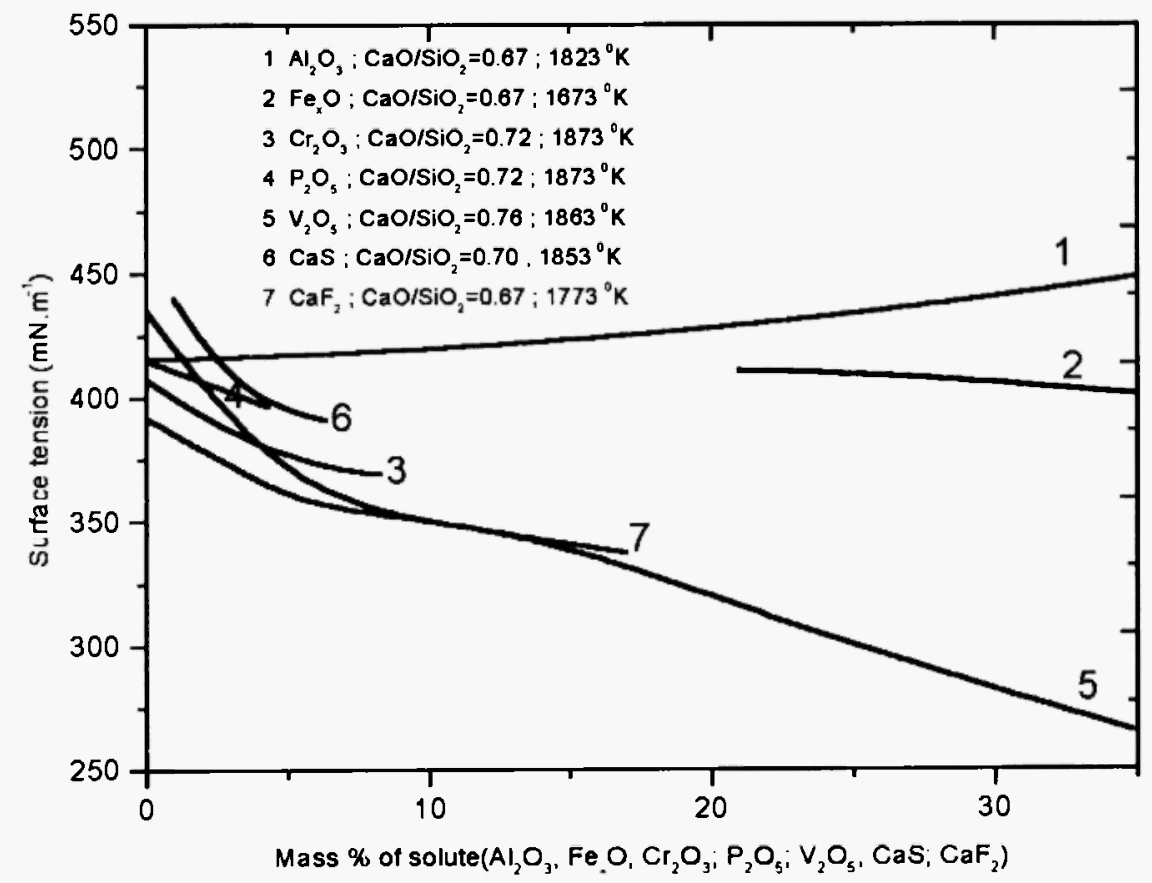

Fig. 1: Iffect of different solutes on the surface tension of $\mathrm{SiO}_{2}-\mathrm{CaO}$ system $/ 20$. 


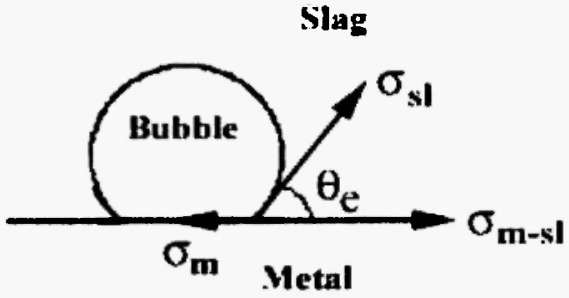

Fig. 2: Schematic diagram of gas-slag-metal interface.

surface tension of slag and interfacial tension between slag and metal $123,24 \%$ Since, at the gas-slag-metal interface, Fig. 2, the equilibrium contact angle, $\theta_{\mathfrak{r}}$, and surface tensions are related by:

$$
\cos \theta_{\mathrm{e}}=\left(\sigma_{\mathrm{m}}-\sigma_{\mathrm{m}-\mathrm{sl}}\right) / \sigma_{\mathrm{sl}}
$$

lower slag-metal interfacial tension. $\sigma_{m-s i}$, and slag surface tension, $\sigma_{\mathrm{sl}}$, result in a low contact angle. Thereby, the presence of surface active compounds in slag produces lower bubble size and stable foam.

Eq. 2 shows that foaming index is directly proportional to slag viscosity. It is well known that the viscosity of slag is primarily determined by the relative amounts of network formers and breakers. amount of dispersed solid phase and the liquidus temperature of slag. Both network former and dispersed solid phase increase the slag viscosity so these slags have higher foaming index.

\section{SOLID ADDITIVES}

When a bubble comes in contact with a solid surface, the instantaneous contact angle differs from the equilibrium value. So the horizontal component of surface tension forces along the line of contact of three phases are not balanced and this results in a net force. Fig. 3 shows the surface tension forces acting along the contact line for a bubble where the equilibrium contact angle is obtuse and dynamic contact angle is acute. From Fig. 3(a), when $\theta<\theta$ e, the net outward force is:

$$
\mathrm{F}=\sigma_{\mathrm{S}-\mathrm{sl}}+\sigma_{\mathrm{sl}} \cos \theta-\sigma_{\mathrm{S}}
$$

and at equilibrium,

$$
\sigma_{\mathrm{S}-\mathrm{sl}}=\sigma_{\mathrm{S}}+\sigma_{\mathrm{sl}} \cos \left(180-\theta_{\mathrm{c}}\right)
$$

where $\theta_{\mathrm{e}}$ is the equilibrium contact angle. From Eq. 5 and 6 .

$$
F=\sigma_{\mathrm{sl}}\left[\cos \left(180-\theta_{\mathrm{e}}\right)+\cos \theta\right]
$$

Obviously, a large value of $\theta_{\mathrm{c}}$ and a small value of $\theta$ give rise to a large outward force, which will pull the bubble and try to make it flat. On the other hand, if $\theta>$ $\theta_{\mathrm{v}}$, the force is inward which will try to make the gas solid contact area smaller.

The spreading characteristics of bubbles were found for air-water-glass and air-water-wax system. These two systems were chosen since the contact angles between water and glass and water and wax are respectively $14^{\prime \prime}$ and $109^{\circ}$. The bubbles were produced by injecting air through a nozzle. The bubble sizes were varied by changing the nozzle diameter. The bubbles were followed by video recording. Fig. 4 shows the spreading of a bubble at wax-water interface. The extent and speed of spreading on a wax surface was found to depend on the size of bubbles. Small bubbles did not spread while

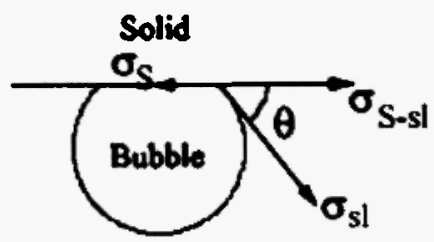

Slag

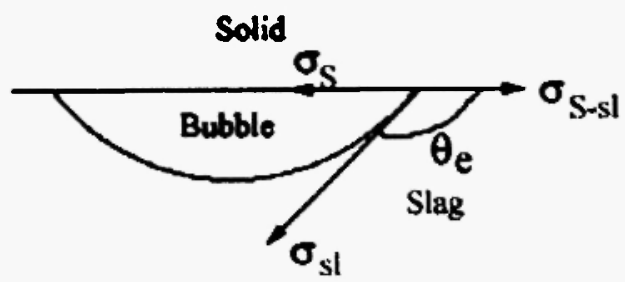

(b)

Fig. 3: Schematic diagram of the forces acting on a bubble in contact with non-wetting surface (a) instantaneous contact angle (b) equilibrium contact angle. 


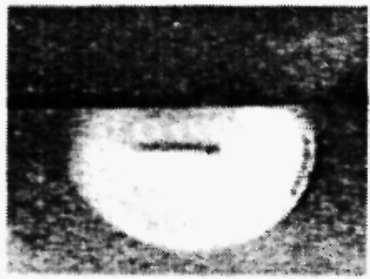

Frame No. $=0$

Time $=0 \mathrm{~s}$

Contact area $=20.0 \mathrm{~mm}^{2}$

Contact angle $=63^{\circ}$

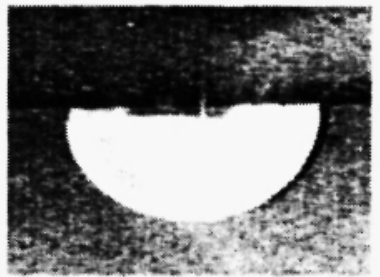

Frame No. $=8$

Time $=0.32 \mathrm{~s}$

Contact area $=30.1 \mathrm{~mm}^{2}$

Contact angle $=102^{\circ}$
Fig. 4: Spreading of bubble at water-wax interface.

very large ones became flatter very fast. Although the exact reason for the observed spreading characteristics has not been ascertained, three factors appear to be responsible. The total driving force for the spreading is proportional to the length of three phase contact line, thereby driving force increases with the increase of the size of the bubble. Secondly, gravity has little effect on the small bubble but it has a significant effect on the shape of large bubbles. Finally, when a bubble comes in contact with wax substrate, the instantaneous contact angle for small bubbles was found to be larger compared that for big bubbles. For a bubble of $2.2 \mathrm{~mm}$ diameter on a wax surface the contact angle was found to be $81^{\circ}$, which did not change, but for a bubble diameter $4.8 \mathrm{~mm}$, shown in Fig. 4 , initial contact angle was $63^{\circ}$. On the glass surface, contact area did not change with time. The contact angle for the glass water system was found to vary between $36^{\circ}-38^{\circ}$ so, according to Eq. 7, the contact line should move inward, but no movement was observed. This supports the view that a minimum force is required for the motion of interface.

Based on the above results, the behaviour of foamy slag in contact with wetting and non wetting solids as observed by Ogawa et al. /14,15/ and Zhang and Fruehan $/ 16 /$ can be explained. Fig. 5 shows the mechanism schematically. When a large bubble comes in contact with the surface of nonwetting solids like carbonaceous material, Fig. 5(a), it spreads over it due to the outward pull along the contact line, Fig. 5(b). This flattening of the bubble pulls the foam upward bringing more bubbles close to the solid surface. When the volume of gas in the film becomes sufficiently large, it comes out from the top of the particle as a large bubble Fig. 5(c) and brings other bubbles in contact with the solid surface. So the net effect is the collapse or rupture of foam. This mechanism of foam collapse was observed by Ogawa et al. /14,15/. If the shape of carbon is spherical as was used by Zhang and Fruehan /16/, the outward pull along the contact line pulls the bubble up giving an appearance of bubble engulfing of the particle, and finally the bubble escapes from the top of

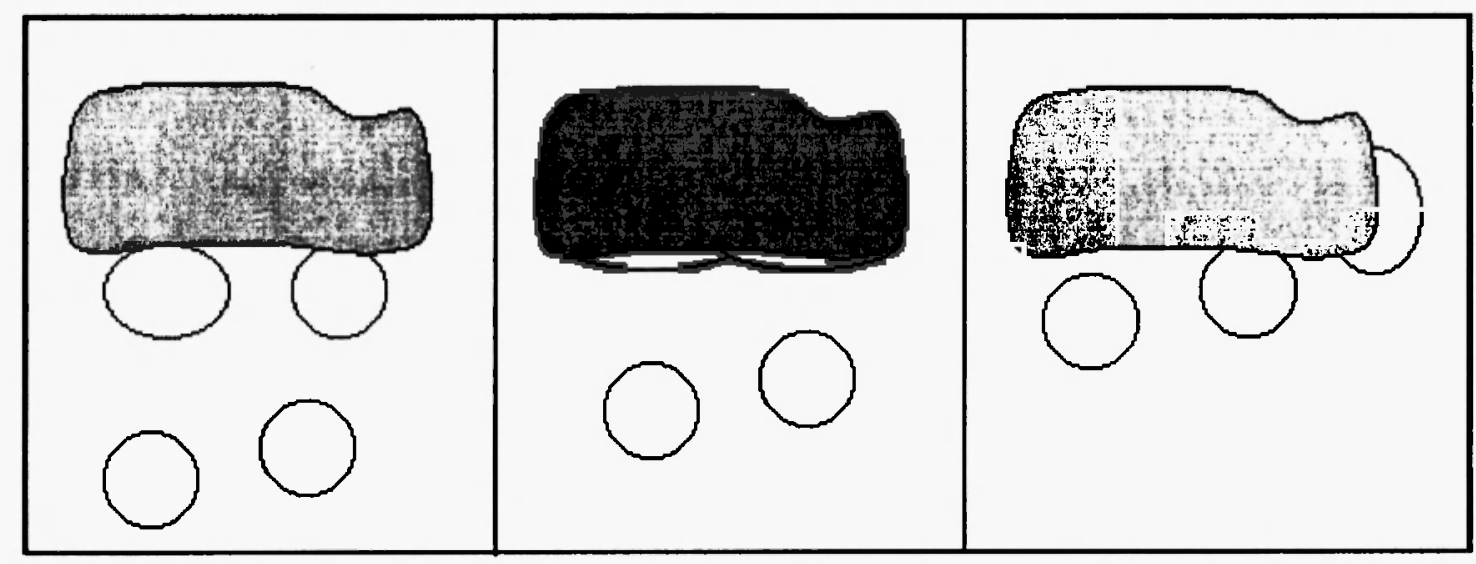

(a)

(b)

(c)

Fig. 5: Schematic diagram of mechanism of bubble rupture on graphite block (a) at time $t_{1}$ (b) at time $t_{2}$ (c) at time $t_{\text {.. }}$ where $t_{3} \cdot t_{2}>t_{1}$. 


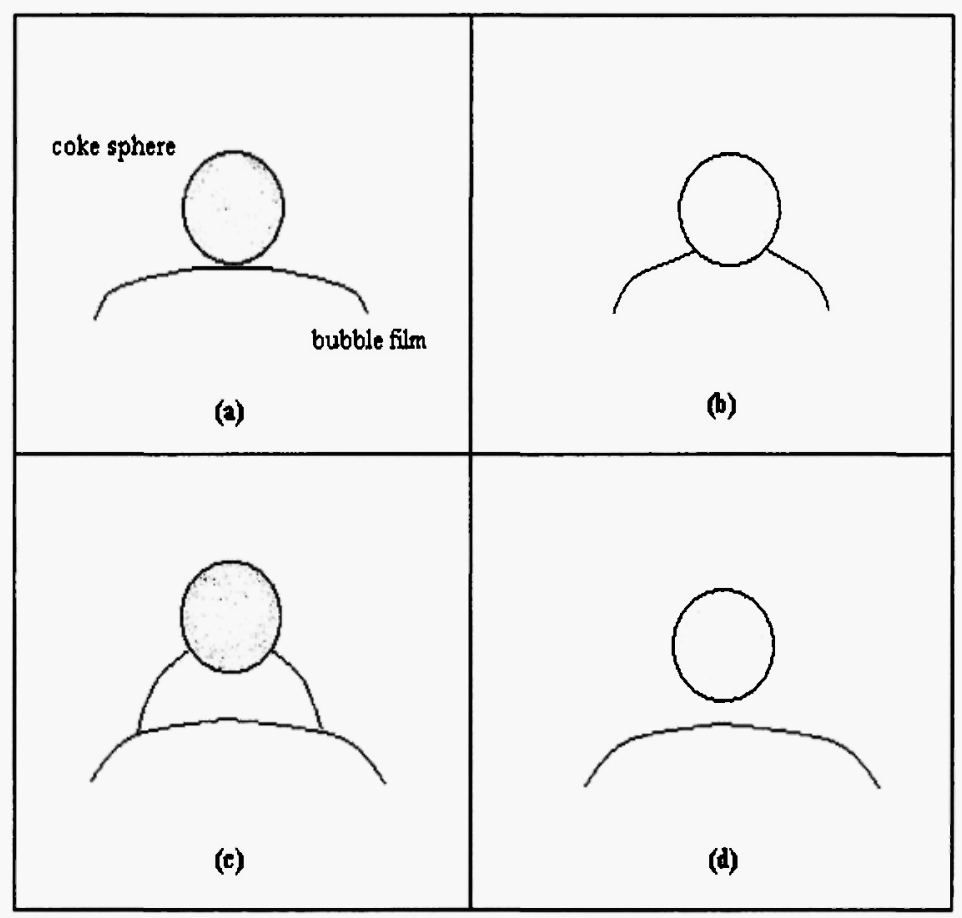

Fig. 6: Schematic diagram of mechanism of bubble rupture on a coke sphere observed by Zhang and Fruehen $/ 16 /$.

the particle. Fig. 6 schematically shows the observations of Zhang and Fruehan 16\%. The upward pull of the bubble brings more of the bubble in contact with the particle and this lead to foam collapse. Obviously wetting solids like alumina or iron oxide has no effect on the bubble collapse.

Ogawa and Tokumitsu /14/ found that a glassy carbon block was not effective to reduce the foam height when bubbles were small and the carbon block was stationary. But when the carbon block was moved up and down, a big bubble was evolved. When a small bubble comes in contact with carbon block, it does not flatten or rupture as was found for air-water-wax system, so there is no pull in the foam and other bubbles do not come in contact with the particle. Therefore there is no foam collapse. But when the carbon block is moved, more bubbles join the attached bubble on the carbon block, increasing its volume, which finally comes up from the surface leading to decrease in foam height.

The mechanism of foam collapse in the presence of carbonaceous particles indicates that if the size of carbon particles is much smaller than the bubbles, it will not be effective. These fines being insoluble in slag increases its viscosity, thereby increasing foam stability according to Eq. 2. This is in agreement with the observations of Wu et al. /18/ who found that the addition of carbon fines smaller than $110 \mu \mathrm{m}$ increased foaming index of slag.

\section{CONCLUSION}

The foaminess of slag increases with increase of $\mathrm{d} \sigma / \mathrm{dc}$ and viscosity. Strong surface active compounds make the foam stable by producing smaller bubbles and making them less prone to rupture.

When bubbles come in contact with non-wetting surfaces, the difference between the dynamic contact angle and equilibrium contact angle pulls a three phase contact line in the outward direction. This force makes the large bubbles flat and results in foam collapse. This force is not significant for small bubbles and is not effective when non-wetting particles are much smaller than the bubbles. 


\section{REFERENCES}

1. H. Katayama, T. Ohno, M. Yamauchi, M. Matsuo, T. Kawamura and T. Ibaraki: ISIJ Int., 32, 95-101 (1992).

2. K. Lahiri: Proc. Workshop Prod Liq. Iron using Coal, Regional Research Lab, Bhubaneswar, India, 149-153 (1994)..

3. Di Donato, G. Malgarini, F. Oeters and L. Zhang: Steel Research, 70, 386-394 (1999).

4. R. Diao: Iron and Steel (Peking). 34, 12-14 (1999).

5. F. Cooper and J. A. Kitcher: JISI, 193, 48-55 (1959).

6. J. H. Swisher and C. L. McCabe: Trans TMSAIME, 230, 1669-75 (1964).

7. S. Hara, M. Ikuta, M. Kitamura and M. Ogino: Tetsu-to-Hagane, 69, I152-59 (1983).

8. K. Ito and R. J. Fruehan: Metall. Trans. B, 20B, 509-514 (1989).

9. R. Jiang and R. J. Fruhen: ibid, 22B, 481-489 (1991).

10. R. E. Roth. R. Jiang. R. J. Fruehan: Trans. ISS, 19 , Nov., 55-63 (1992).

11. Y. Zhang and R. J. Fruehan: Metall. and Mater. Trans B, 26B. 803-812 (1995).

12. S. Jung and R. J. Fruehan: ISlJ Int., 40, 348-355 (2000).
13. K. Lahiri and S. Seetharaman: Met. and Mater. Trans. 33B, 499-502 (2002).

14. Y. Ogawa and N. Tokumitsu: Proc. Sixth International Iron and Steel congress, Nagoya. ISIJ, 147-152 (1990).

15. Y. Ogawa. H. Katayama, H. Hirata, N. Tokumitsu. M. Yamauchi: ISIJ Int., 32, 87-94 (1992).

16. Y. Zhang and R. J. Fruehan: Metall. Mat. Trans. B. 26B, 813-819 (1995).

17. Dippenaar : Int. J. Miner, Proc., 9, I-22 (1982).

18. K. Wu, W. Qian, S. Chu, Q. Niu and H. Luo: ISIJ Int., 40, 954-957 (2000)

19. S. Sun Nexhip and S. Jahanshai: Metall. and Mater. Trans B. 31B. 1105-1115 (2000).

20. Slag Atlas, $2^{\text {nd }}$ Edition, Edited by VDEh, Verlag Stahleisen GmbH, 430-44I (1995).

21. S. Hara, M. Kitamura and K. Ogino: ISIJ Int., 30. 714-721 (1990).

22. O. Ogawa, D. Huin, H. Gaye and N. Tokumitsu: ISIJ Int., 33, 224-232 (1993).

23. K. Nakashima and K. Mori: ISIJ Int., 32. II-18 (1992)

24. T. El Gammal and P. Stracke: Proc: $3^{\text {int }}$ Int. Conf. On Molten Slags and Fluxes, Glasgow, June 1988. Institute of Metals, London, 51 (1989). 
